\title{
Téoros
}

Revue de recherche en tourisme

\section{La Route verte, un circuit touristique ?}

\section{Sylvain Lefebvre et Maryse Trudeau}

Volume 22, numéro 2, été 2003

Les Routes à thèmes

URI : https://id.erudit.org/iderudit/1071596ar

DOI : https://doi.org/10.7202/1071596ar

Aller au sommaire du numéro

Éditeur(s)

Université du Québec à Montréal

ISSN

0712-8657 (imprimé)

1923-2705 (numérique)

Découvrir la revue

Citer cet article

Lefebvre, S. \& Trudeau, M. (2003). La Route verte, un circuit touristique ?

Téoros, 22(2), 46-51. https://doi.org/10.7202/1071596ar d'utilisation que vous pouvez consulter en ligne.

https://apropos.erudit.org/fr/usagers/politique-dutilisation/ 


\section{La Route verte, un circuit touristique'?}

\section{Sylvain Lefebvre et Maryse Trudeau}

En Amérique du Nord, il aura fallu près de trois décennies pour confirmer le vélo comme usage récréatif et moyen de transport populaire dans certaines villes. Le Québec a sans aucun doute fait ses classes et assuré une place de choix à l'usage généralisé de la bicyclette. Mais, il n'y a pas si longtemps, l'infrastructure cyclable québécoise était encore très désuète. Les cyclistes et leurs organisations ont milité pendant de nombreuses années pour la mise en place de voies praticables et sécuritaires. Des aménagements ont ainsi été réalisés au cours des années 1970 et 1980 et d'autres voient encore le jour aujourd'hui (Jolicoeur, 2001). Le projet de la Route verte puise ses racines dans cette demande récurrente d'un réseau convenable pour circuler à vélo.

Qu'est-ce que la Route verte? La Route verte est en réalité un itinéraire cyclable de plus de 4000 kilomètres qui sillonne le Québec du nord au sud et de l'est à l'ouest. Elle traverse villes et villages, longe rivières et plans d'eau, explore les sites naturels et culturels. C'est un moyen de découvrir le Québec en traversant campagnes et villes. Le trajet est composé de six axes principaux:

- l'axe 1 va de Hull à Gaspé en bifurquant vers Sherbrooke pour longer la Rive-Sud du fleuve à partir de Québec ;

- l'axe 2 commence à Ville-Marie en Abitibi-Témiscamingue, traverse les Laurentides pour se raccorder aux ÉtatsUnis via la vallée de la rivière Richelieu ;
- l'axe 4 prend sa source dans le parc de la Mauricie pour traverser le Centre-duQuébec et l'Estrie jusqu'à la frontière américaine ;

- l'axe 5 commence à Rivière-Baudette, à l'ouest de Montréal et suit la Rive-Nord du fleuve pour s'arrêter à Québec ;

- l'axe 6 se prolonge de Rivière-à-Pierre au nord de Québec jusqu'en Beauce ;

- L'axe 8 fait le tour du Lac-Saint-Jean, longe le Saguenay, enjambe le fleuve et traverse le Bas-Saint-Laurent pour se joindre au réseau du NouveauBrunswick.

Le trajet de la Route verte est circonscrit à l'écoumène québécois. Elle rejoint plus de la moitié de la population québécoise, soit près de quatre millions de personnes (Vélo Québec, 2003). Certains itinéraires d'Europe, tels que le projet du National Cycle Network au Royaume-Uni et le projet RAVeL en Belgique, tentent également de rejoindre une grande partie de la population (Maréchal et Daubechies, 1999). La caractéristique principale qui différentie ces itinéraires de la Route verte est la densité des voies cyclables qui, dans ces pays, s'organisent en un réseau qui ratisse systématiquement le territoire.

L'idée de bâtir un itinéraire qui permet de parcourir le Québec d'un bout à l'autre est intéressante, mais la grande superficie couverte et la répartition inégale de la population ont pour effet de produire un réseau asymétrique, long et parfois monotone. La traversée de l'Abitibi ou de la Gaspésie se traduit par un grand nombre de kilomètres et par une offre réduite en services et attraits. Plusieurs questions intéressantes émergent si l'on regarde de près l'organisation territoriale de ce réseau. La Route verte est-elle un véritable circuit touristique? Pour desservir quelle clientèle? Rejoint-elle les besoins des cyclistes qué-

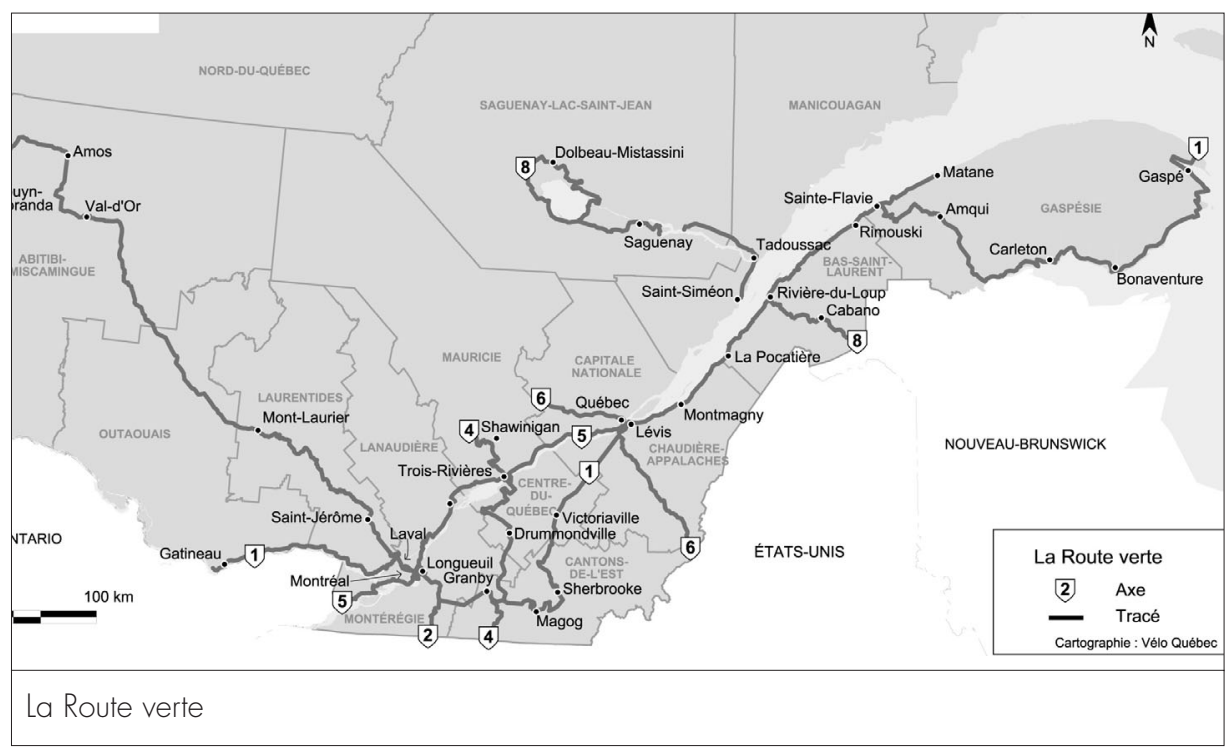




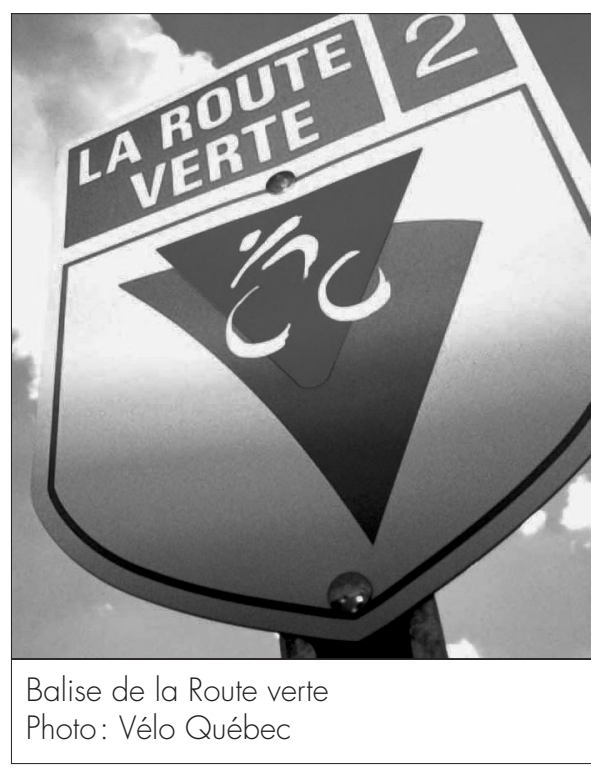

bécois? N'aurait-il pas été justifié, par exemple, de concentrer les efforts d'aménagement dans les régions centrales afin de construire un réseau dense de voies cyclables capables de satisfaire un plus grand nombre de cyclistes plutôt que d'accommoder des cyclotouristes d'expérience?

La Route verte n'est pas le premier projet visant à construire un long itinéraire multifonctionnel. En effet, elle a puisé son inspiration de diverses expériences. Les grands itinéraires cyclables du monde tels que la Cycloroute nationale du Danemark, les pistes cyclables du Danube et du Rhin et l'itinéraire côtier de l'Oregon et de la Californie, ont, par leur essor et leur développement, insufflé un élan de créativité au projet québécois (Jolicoeur, 1997). À noter également que cet itinéraire est de la même lignée que les grands sentiers multifonctionnels du Canada (Pronovost et al., 1999).

\section{Le concept}

Le concept de la Route verte s'appuie sur certains fondements qui lui donnent son caractère particulier. Premièrement, elle se veut un moyen sécuritaire de circuler à vélo pour une majorité de cyclistes. Les types d'aménagements cyclables choisis visent à assurer un maximum de sécurité. Le deuxième fondement provient du type de tourisme dont elle s'inspire. Le cyclotou- risme est une forme de tourisme d'aventure douce. L'effort physique, le contact avec la nature, le respect de l'environnement sont donc des éléments intégrés à la Route verte. Le troisième fondement est le caractère rassembleur du projet. La mise en place de voies cyclables formant une route qui traverse la province exige une collaboration étroite entre une multitude de partenaires. Cette route est plus qu'un simple parcours, elle est un élément catalyseur des forces locales, régionales et nationales (Jolicoeur, 1997: 14).

Certains principes guident l'aménagement de la Route verte. La création d'une unité d'ensemble réunissant tous les tronçons du projet est la base même du concept. Les tronçons s'unissent donc en adhérant à un nom et à un logo identiques, tout en conservant leur appellation d'origine. Cette duplication, bien qu'ambiguë, permet la commercialisation de chaque tronçon à différentes échelles. Selon le principe de l'accessibilité, la route doit être ouverte à tous, en tout temps: les cyclistes, les marcheurs et les cavaliers sont admis sur le tracé, mais l'accès est interdit à tout véhicule motorisé. La période d'ouverture s'étend d'avril à octobre, en fonction des conditions climatiques. Vélo Québec, principal architecte du réseau, favorise également la gratuité d'accès. Un troisième principe encourage une signalisation normalisée sur l'ensemble du réseau, adaptée à la clientèle. Le dernier principe est celui de la permanence: le tracé de la Route verte doit être assuré d'une certaine pérennité : continuité dans l'espace, en permettant aux gens de se retrouver et de reconnaître le tracé et les infrastructures, et dans le temps, en inscrivant le tracé dans les schémas d'aménagement et les plans de zonage et en confiant la gestion à des organismes stables.

Le projet de la Route verte s'appuie aussi sur la collaboration entre les nombreux intervenants qui visent la consolidation du réseau cyclable québécois. Pour bâtir la route, il a fallu «utiliser les infrastructures en place et susciter le développement de nouveaux axes dans les régions où de tels équipements (étaient) inexistants» (Archambault et al.,1997:11). Le projet de la Route verte a pris comme base les divers projets d'aménagement cyclable, en cours de réalisation ou à l'étude, au Québec. La piste du canal de Chambly, le lien entre Oka et Deux-Montagnes et le tour du LacSaint-Jean en sont d'excellents exemples.

Fruits d'un travail conjoint entre régions, municipalités et groupes de citoyens, les pistes cyclables ont été réunies sous la bannière de la Route verte. C'est donc davantage un effort de consolidation des projets existants et en voie de réalisation qui a été fait dans la mise en place de la Route verte, plutôt qu'un effort de planification intégrée voulant déterminer la configuration optimale d'un tel tracé sur le territoire québécois.

La Route verte est un projet en évolution. Compte tenu de son ampleur, les intervenants proviennent des différentes échelles gouvernementales et administratives. Chacun des paliers a un rôle à jouer. Le ministère des Transports du Québec (MTQ) fournit l'expertise en matière d'aménagement, les municipalités s'occupent de l'acquisition d'emprises, les gestionnaires locaux de l'entretien et Vélo Québec de la concertation (Panneton, 1999). Le projet concerne également des centres de développement économique, des organismes sans but lucratif et des entreprises privées. Pour le moment, il n'y a que le volet touristique qui n'a pas de véritable partenaire associé. La création d'une route cyclable n'entraînera pas d'elle-même la mise en place de services et d'attraits pour les visiteurs à vélo. Le développement de l'offre par les localités doit être stimulé par des subventions et appuyé par une expertise, d'où l'importance de s'associer des intervenants du milieu touristique.

L'expression «Route verte» suggère l'image d'une route continue où paysages et infrastructures du réseau s'harmonisent, où les attraits et les services sont disponibles et équitablement répartis sur l'ensemble du territoire, où enfin l'expérience cyclable apparait diversifiée et polyvalente. Est-ce la réalité ? Peut-on parler ici de circuit ou d'itinéraire touristique? Qu'estce que la Route verte? Quelle est sa véritable nature? 


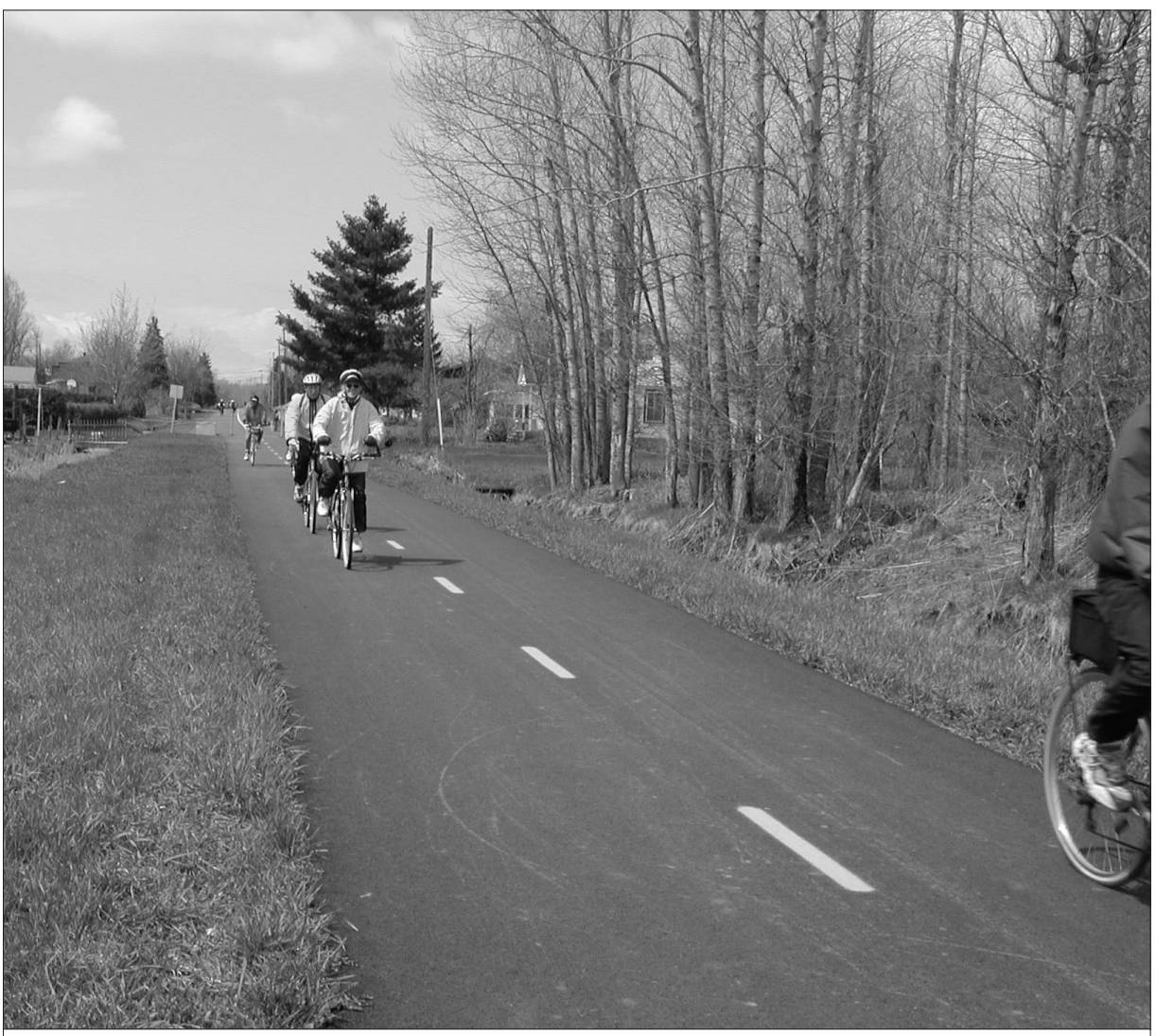

Piste cyclable de Carignan (inauguré en 2002) nommée Montée du Fort-Chambly Photo: Vélo Québec

La Route verte n'est pas une route touristique à thème. Elle en possède bien certaines caractéristiques, mais le concept même de ce projet va à l'encontre de son nom. Tout d'abord, la Route verte est divisée en six routes différentes qui sont qualifiées «d'axes». Le projet a été pensé et aménagé non pas comme une seule route, mais comme un croisement de plusieurs tronçons.

Un itinéraire, une route ou un circuit touristique, c'est d'abord l'assemblage de cinq composantes: le tracé, les services, les attraits, la communication et le thème (Trudeau, 2002 : 42). La présence simultanée de ces cinq ingrédients a pour but de former un tracé cohérent, fluide et continu et même si chacune des composantes peut être présentée individuellement, seule leur combinaison caractérise une véritable route touristique. La Route verte n'est pas encore un circuit touristique au sens propre du terme et ne sera peut-être jamais vraiment amenée à le devenir. L'inauguration et l'achèvement des travaux étant prévus pour 2005-2006, il serait hasardeux de se prononcer sur sa forme finale.

\section{Le tracé}

La Route verte se déploie dans seize régions administratives et parcourt des ensembles géographiques très diversifiés. Certains tronçons, semblables d'un point de vue morphologique, génèrent naturellement des ensembles homogènes où règnent une certaine harmonie entre les éléments, une continuité et une fluidité dans les paysages. Ces ensembles homogènes constituent l'attrait principal des voyageurs à vélo. Ce sont eux qui colorent le trajet cyclable, qui rythment le voyage et déterminent l'esprit dans lequel se trouve le voyageur. Une combinaison trop audacieuse de différents paysages pourrait dérouter le cycliste et le retirer de l'espace mental de voyage dans lequel il se trouve. En plus de traverser différents espaces physiques (zones maritimes, zones forestières, etc.), la Route verte traverse des régions où la distribution spatiale de la po- pulation varie considérablement. On le sait, le Québec est inégalement peuplé. La moitié de la population québécoise réside dans la grande zone urbaine de Montréal alors que l'autre moitié se disperse sur l'ensemble du territoire et les routes touristiques sont influencées par cette organisation spatiale de la population. L'étalement des villages et la densité du tissu urbain jouent un rôle important dans la cadence du voyage. La fréquence d'enchaînement des intersections, des voies cyclables, des paysages, des villes et des villages modifie la vitesse à laquelle semble se dérouler le voyage. Plus le décor change autour du cycliste, plus vite est le mouvement. Ce phénomène a pour effet d'accélérer la cadence. De même, la vitesse et le cycle de passage des voitures imposent leur rythme aux cyclistes. La Route verte circulant à travers villes et campagnes propose ainsi divers rythmes de déplacement.

La Route verte ne se compose pas que de routes touristiques ; il existe en effet des portions de tracé qui ne possèdent pas suffisamment de « charisme » ou de «personnalité » pour être élevées au rang de route. L'espace dans lequel ces tracés sont implantés ne possède pas de caractère particulier : le paysage est transitoire, voire monotone ; les villages rencontrés sont dépourvus de charme et l'offre en services est rare. Ce ne sont en réalité que des liens entre deux portions de routes qui présentent davantage d'intérêt et ces liaisons ont pour effet de réduire l'enthousiasme des cyclotouristes.

Pour bien appréhender la Route verte, il faut considérer aussi les portions qui seront achevées dans les prochains mois, portions ne sont pas exactement des routes, mais détiennent un potentiel pour le devenir. Le long de ces tronçons, le paysage est parfois agréable et cohérent, les services sont présents quoique peu nombreux et dispersés. On ne peut leur attribuer le nom de route touristique, car ces atouts ne sont pas encore mis en valeur pour les usagers. Les gens du milieu n'ont pas pris possession de cette infrastructure et n'ont, par le fait même, pas donné vie au tracé. L'appropriation régionale fait donc défaut à ces tronçons en devenir. 
Qu'est-ce qui permet d'affirmer que certains tronçons méritent plus le label de « route touristique » que la Route verte ellemême ? D'abord, ils possèdent un début et une fin. En cela, ils n'offrent pas la liberté de choisir entre différents itinéraires comme le fait la Route verte avec ses axes. De plus, ils sont ceinturés par des espaces géographiques et humains semblables. Le type de paysage, le type de voies cyclables et l'étalement des villes et des villages forment un espace géographique au cœur duquel la route prend place. Elle s'imprègne alors de l'environnement qui l'entoure et forge sa personnalité.

\section{Les services et les attraits}

La quantité et la fréquence des services offerts aux cyclotouristes fluctuent beaucoup tout au long de la Route verte. Certaines portions du trajet, comme la section sud de la piste du Parc linéaire du P'tit Train du Nord ou la Véloroute des Bleuets, offrent aux cyclistes une quantité importante de services répartis sur l'ensemble du réseau. À l'opposé, des régions touristiques telles que l'Abitibi-Témiscamingue ou Lanaudière disposent de peu de services qui, d'ailleurs, sont dispersés. La Route verte est donc segmentée ; elle ne forme pas un tout en fonction de l'organisation spatiale et de la quantité de l'offre. D'ailleurs, l'offre influence le type de clientèle qui parcourt la piste : par exemple, une famille recherchera un trajet où les services sont fréquents, tandis qu'un club cycliste optera pour la qualité des services. Les gestionnaires de pistes aménagent habituellement leur produit en fonction de la clientèle cible. Le type de voies cyclables est choisi, les haltes sont positionnées et des stationnements sont aménagés. Chacune des clientèles a des besoins particuliers et ce ne sont pas toutes les portions du réseau qui peuvent les combler. En ce sens, la Route verte ne peut être parcourue par la même clientèle d'un bout à l'autre du réseau ; elle est davantage sillonnée par sections, par spécialisations.

\section{La communication}

La Route verte est tracée, aménagée et gérée localement. Dans certains cas, ce sont des organismes privés qui administrent les portions de route; dans d'autres, ce sont les MRC, les villes et les régions administratives, alors que le ministère des Transports du Québec veille à l'entretien de l'équipement sur son territoire. Il existe donc une multitude d'acteurs impliqués dans le projet. Ces derniers, bien que régis par des règles, développent leur portion selon leurs désirs. Ils aménagent la piste en fonction de la clientèle qu'ils ciblent, du thème et d'un label distinctif. Un itinéraire pourra ainsi être parsemé d'anciennes gares de chemin de fer témoins de l'histoire passée, tandis qu'un autre traversera vergers et vignobles. Dans un autre cas, c'est le paysage qui traduira l'esprit des lieux. Les gestionnaires personnalisent leur tronçon. Malgré tous ces éléments qui divisent, découpent, segmentent la Route verte en tronçons, le réseau global diffuse une image qui, elle, se veut unificatrice.

La Route verte est partiellement standardisée par des moyens de communication. La signalisation sur l'ensemble des tronçons est uniforme. Elle respecte les règles établies, identifiant au cycliste qu'il est au bon endroit. Contrairement à la mise en marché des régions touristiques du Québec, la Route verte rassemble les différentes pistes cyclables en donnant une image unifiée du produit cyclable dans la province. Ce regroupement facilite la mise en vente de la route comme un ensemble et donne la liberté aux touristes de choisir leur destination cyclable, sachant où ils peuvent trouver un itinéraire. Les normes de construction et d'entretien rigoureuses visent à mettre en place des infrastructures de qualité qui demeureront en place longtemps. Ces normes contribuent à une certaine uniformisation du réseau.

La Route verte devra vraisemblablement mettre sur place une offre touristique pour combler les besoins diversifiés de la clientèle cycliste. Les divers ouvrages ou lieux où l'on vend la Route verte doivent mettre à la disposition des lecteurs des parcours, des services et des attraits. Vélo Québec publie des ouvrages sur le réseau et investit progressivement dans le volet touristique du projet. L'ajout de ces renseignements permettra aux cyclistes de construire leur propre itinéraire. Dans la troisième édition du Guide de la Route verte, se trouve une liste de lieux d'hébergement et d'attraits susceptibles de plaire à la clientèle cycliste. Cet ajout d'information est intéressant, mais gagnerait à être davantage approfondi. Une structure de l'offre touristique doit être montée. Les développeurs de la Route verte doivent réfléchir aux types d'hébergement qu'ils veulent inclure à leur offre, aux types d'attraits qu'ils veulent proposer et aux parcours qu'ils veulent suggérer. La question de la restauration demande aussi à être poussée davantage. Doivent-ils ne mentionner que les services bicycle friendly ou simplement souligner ceux qui sont à proximité du tracé? Il est important, pour identifier l'information qui doit faire partie de la promotion de la Route verte, de faire une étude et d'élaborer une liste de critères de sélection en fonction de l'image que l'on désire véhiculer.

\section{Le thème}

Le thème est également une composante essentielle d'une route touristique. La découverte du Québec à vélo demeure le thème principal, mais l'application d'un sujet particulier à une piste ou à un tronçon relève sa singularité. Des thèmes tels que le chemin de fer, le littoral, la montagne sont révélateurs du paysage et de l'environnement dans lequel circule la piste; Ils éveillent chez le cycliste le désir de les parcourir. En plus du thème, l'attribution d'un nom significatif à un tronçon octroie à ce dernier une identité. Il passe alors du statut de portion à celui de route; il prend vie. En résumé, les routes touristiques doivent être dotées d'une personnalité.

La motivation du voyage peut se traduire par l'exploration d'une région, l'approfondissement des connaissances, le voyage dans le temps ou la visite d'une attraction particulière. Dans le cas de la Route verte, c'est la découverte des différents visages du Québec en vélo qui motive les déplacements. Que l'on parcoure la Gaspésie, les Laurentides ou le Centre-du-Québec, l'objectif est de découvrir des lieux et de s'en imprégner. L'avantage d'emprunter la 


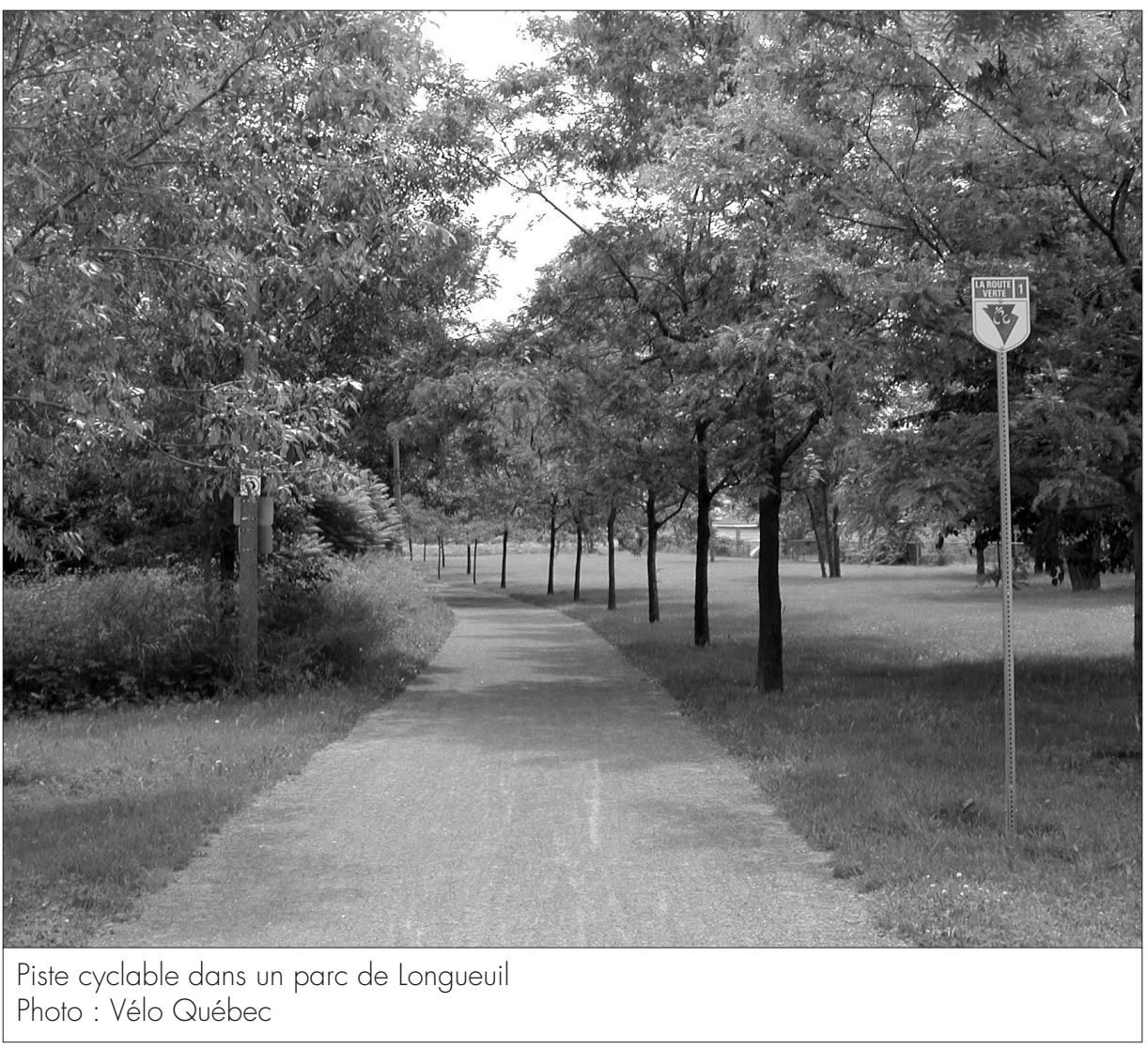

Route verte est que le vélo offre une cadence ralentie qui permet le contact avec l'environnement.

La Route verte regroupe plusieurs routes. Ces dernières sont à des stades de développement différent, possèdent une force attractive distincte et une popularité inégale. Elles sont uniques. Par exemple, le Parc linéaire du P'tit Train du Nord se situe dans un paysage montagneux parsemé de lacs et de rivières où les villages se succèdent. Le thème de la route a été puisé à même l'emplacement de la piste. Aménagée sur l'emprise d'un ancien chemin de fer, la voie cyclable fait revivre l'époque du développement de la région. Elle porte d'ailleurs le nom du train qui lui a donné naissance.

Un autre tronçon imposant est celui qui longe le fleuve Saint-Laurent. Cette portion possède une dynamique totalement différente du cas précédent. La Route verte se donne un visage de tranquillité baignée par une masse d'eau d'un côté et les collines de l'autre. La multitude de petits villages assure aux cyclotouristes une offre en services présente quoique clairsemée. Toutefois, nous ne pouvons parler à ce stade de route touristique. Tout d'abord, cette portion n'est pas achevée. De plus, aucun organisme n'a pris en charge ce tronçon et elle n'a pas été identifiée comme route ; aucun thème ou nom ne lui a été donné. Elle n'a d'ailleurs pas réellement de début et de fin. Un des défis de la consolidation de la Route verte réside dans la création de tronçons pour les segments qui se retrouvent dans un ensemble géographique et humain significatif.

La Route verte n'est pas une route touristique à thème, non pas parce qu'elle ne possède pas le potentiel requis, mais parce que l'agencement des différentes composantes du circuit touristique n'est pas cohérent en soi. Les tronçons qui la constituent possèdent, dans le cas de certaines routes touristiques, une personnalité plus forte que la Route verte elle-même. Cette dernière n'a utilisé, d'être une route touristique. Son rôle est davantage de combiner les routes cyclables afin d'offrir aux cyclistes des trajets plus longs et de donner de la force au prod'ailleurs pas la prétention, malgré le label duit touristique «vélo». L'objectif de la Route verte, ce n'est pas d'être parcourue en un seul voyage, mais d'être roulée par tronçons.

La mise en marché de la Route verte comme un tout cohérent n'a pas été optimale au cours des dernières années. En effet, la promotion de La Route verte passe par la mise en valeur des axes décrits plus haut et, ce faisant, diffuse une image plutôt terne du parcours. Cette succession de voies cyclables est divisée en axes numérotés selon leur orientation: impairs de l'ouest vers l'est et pairs du nord au sud. Cette division rappelle la structure d'un réseau routier. La Route verte n'est cependant pas un réseau de voies cyclables servant principalement aux déplacements utilitaires, mais plutôt un réseau cyclotouristique. Elle doit donc être attractive si elle veut être parcourue par des touristes. L'attribution d'une signature propre à l'environnement concerné attire la clientèle davantage qu'un numéro. La Route verte aurait été plus vivante si le découpage s'était appuyé sur les spécificités territoriales et l'environnement physique plutôt que sur l'orientation géographique du tracé et si des noms avaient été accordés à ces mêmes divisions. En effet, nommer une route lui confère un caractère. Quand une portion de route est nommée, cette dernière prend place dans l'espace. De plus, on lui rattache des événements qui y ont eu lieu et on la décrit même par l'environnement qui l'entoure. Par exemple, l'autoroute 40 est aussi appelée «Transcanadienne». Ce nom indique clairement que cette route traverse le Canada. Même si cette information demeure triviale, elle reste tout de même suffisante pour lui octroyer un caractère particulier. De même, l'autoroute 15 Nord est appelée «Autoroute des Laurentides», car elle traverse cette section du Bouclier canadien. Ces routes sont bien plus que des numéros; elles ont une personnalité et un caractère distinctif.

Outre le sens de la route, quelles images transmettent les axes de la Route verte aux cyclistes? Les axes ne parlent pas, ils ne diffusent aucun indice sur la nature des tronçons, sur les paysages qu'ils traversent, sur ses particularités. La Route verte est un 
équipement touristique intéressant qui offre aux cyclistes un environnement agréable. Elle aurait eu tout à gagner à diviser le Québec selon les grands espaces touristiques et à nommer ces divisions. Par exemple, la portion après Lévis aurait pu se nommer La route du Saint-Laurent et l'agencement de la Véloroute des Bleuets et du Saguenay, simplement Le Tour du lac Saint-Jean et $d u$ fjord du Saguenay. Maintenant que les axes ont été numérotés, le retour en arrière est impossible. La mise en marché peut cependant être modifiée et s'orienter davantage sur la promotion de grands ensembles tels que véhiculés dans la troisième édition du Guide de la Route verte.

\section{Conclusion}

La Route verte n'est pas une route touristique, bien qu'elle en possède certaines composantes. Elle est davantage un assemblage de routes à thème et de liens articulés pour le vélo ; elle propose un rôle de rassembleur et de catalyseur du réseau cyclable québécois, tout en respectant la nature de chacun de ses propres tronçons et en collaborant avec les acteurs concernés. Nous l'avons mentionné, la Route verte n'est pas achevée et ne le sera pas avant 2005-2006. Dans l'état actuel des choses, la Route verte ne possède pas suffisamment de services et d'attraits pour répondre aux besoins de sa clientèle. Nous pouvons présumer qu'avec l'achèvement du projet, l'offre globale sera très intéressante. Cet ajout fera-t-il de la Route verte un véritable circuit touristique? Nous croyons que la diversité des entités qui la composent et la combinaison non structurée de celles-ci ne permettent pas et ne permettront pas, malgré les modifications à venir, de faire de la Route verte un circuit touristique.

Une fois ce constat réalisé, la Route verte doit néanmoins être perçue comme un projet unificateur. On peut y puiser des idées d'itinéraires à l'image du Québec routier qui est une source quasi-inépuisable de circuits touristiques originaux. La Route verte développera vraisemblablement des routes touristiques partielles à même son propre tracé afin de présenter à la clientèle touristique plusieurs itinéraires susceptibles de satisfaire une variété d'usagers. On peut développer des itinéraires d'une journée, d'une fin de semaine ou d'une semaine auxquels se grefferont des services et des attraits parmi lesquels le client pourra faire un choix. Un tel amalgame de routes pourra refléter davantage ce qui semble être la véritable finalité de la Route verte : la diversité dans un tout cohérent. Cet effort de percevoir la Route verte comme un assemblage de routes commence à faire son chemin. En effet, on peut trouver sur le site Internet de la Route verte une série de parcours suggérée à la clientèle. Ces «coups de cœur» ont pour but de faire connaître un tronçon de la Route verte et d'inviter les gens à tenter l'expérience.

De plus, un effort devrait être fait pour pousser les gestionnaires des pistes à développer leur portion de tracé. Une fois la piste construite, l'opération suivante ne se limite pas uniquement à son entretien. Il faut en faire un tronçon agréable à parcourir et possédant un caractère particulier. Les gestionnaires doivent faire de leur portion une véritable route touristique si tel est leur désir; ils doivent lui donner un nom significatif; y aménager des haltes, y offrir des services et des attraits et les intégrer de façon harmonieuse au plan global de la Route verte. Mais avec la configuration du territoire québécois et le morcellement de ses ensembles physiques et humains trop différents les uns des autres pour former un système fluide, la configuration de la Route verte prendra vraisemblablement la forme d'un réseau en arborescence, avec des tronçons très variables les uns des autres notamment en contenu et en services, qui seront soumis à une logique administrative et politique locale/régionale. L'opération n'aura rien perdu de son intérêt en soi, mais elle ne devra pas être perçue ou comprise comme une opération d'aménagement global adapté aux spécificités d'un circuit touristique.

Sylvain Lefebvre est professeur au Département de géographie de l'UQAM et Maryse Trudeau détient une maîtrise en géographie de la même université.

\section{Note}

1 Cet article repose sur les principales conclusions du mémoire de maîtrise en géographie de Maryse Trudeau (2002).

\section{Bibliographie}

Archambault, Michel, Nancy Giguère, Patrick Joly, et Université du Québec à Montréal, Chaire du tourisme (1997), Les retombées économiques de la Route verte: Rapport final présenté à Vélo Québec, Montréal, UQAM, École des sciences de la gestion, Chaire du tourisme.

Jolicoeur, Marc (2001), L'état du vélo au Québec en 2000, Montréal, Vélo Québec.

Jolicoeur, Marc (1997), Guide de réalisation de la Route verte, Montréal, Vélo Québec, Transports Québec.

Maréchal, Luc, et Anne Daubechies (1999), Le programme RAVeL, une infrastructure pour une autre mobilité, Les cahiers de l'urbanisme, $\mathrm{n}^{\circ} 27$, p. 54-60.

Panneton, Marc (1999), La route verte: Un réseau cyclable national pour le Québec, Revue générale des routes, Paris, p. 120-121.

Pronovost, Jean-François, Marc Lusignan, et François Marcil (1995), Les sentiers du $21^{e}$ siècle: planification, design et gestion des sentiers polyvalents, Montréal, Vélo Québec.

Trudeau, Maryse (2002), Évaluation du potentiel touristique de la Route verte comme circuit touristique, mémoire de maîtrise dirigé par Sylvain Lefebvre, Département de géographie, Université du Québec à Montréal, novembre, 233 pages.

Vélo Québec (2003), En route vers 2005: Plan d'action 2003-2004 pour le développement de la Route verte, Montréal, Vélo Québec. 